\title{
Mapa conceitual no ensino presencial ao remoto durante a pandemia do coronavírus: relato de experiência
}

Conceptual map in face-to-face teaching during the coronavirus pandemic: experience report

Mapa conceptual en la enseñanza presencial durante la pandemia de coronavirus: informe de experiência

Recebido: 11/03/2021 | Revisado: 18/03/2021 | Aceito: 19/03/2021 | Publicado: 27/03/2021

Amanda Regina da Silva Góis

ORCID: https://orcid.org/0000-0003-4661-772X Universidade de Pernambuco, Brasil E-mail: amanda.gois@upe.br

Camila Gabriela da Silva Góis

ORCID: https://orcid.org/0000-0002-5106-8705

Universidade Federal de Pernambuco, Brasil

E-mail: gcamilag@gmail.com

Paulo Filipe Candido Barbosa

ORCID: https://orcid.org/0000-0003-0608-1123

Universidade Federal de Pernambuco, Brasil E-mail: pfcb@cin.ufpe.br

\begin{abstract}
Resumo
Objetivo: relatar a experiência da criação de mapa conceitual no ensino presencial ao remoto durante a pandemia do coronavírus. Metodologia: trata-se de um estudo descritivo-explicativo do tipo relato de experiência. Resultados: Tanto no ensino presencial quanto no virtual os alunos demonstraram total interesse e disponibilidade de participar das atividades propostas. Na primeira turma realizada de forma presencial os alunos puderam ampliar seus conhecimentos e integração utilizando ferramentas diversas, estimulando o uso da criatividade através da aplicação de recursos como canetas, tintas, lápis coloridos, promovendo a interação dos alunos que ao final podiam compartilhar seus trabalhos e realizar uma autoavaliação. Nas aulas remotas utilizou-se plataformas virtuais para a realização da atividade. Divididos em grupos os alunos, mesmo de forma remota, puderam exercer o diálogo e integração. Desta maneira, percebeu-se que o uso de mapas conceituais junto com a prática de uma aprendizagem ativa se torna positivo, tanto no ambiente remoto quanto presencial, auxiliando na compreensão de conceitos. Conclusão: o uso do mapa conceitual em conjunto com a aprendizagem ativa auxilia no desenvolvimento de um ambiente educacional, tanto presencial quanto virtual, prazeroso, capaz de promover integração, pensamento crítico e autonomia entre os alunos.
\end{abstract}

Palavras-chave: Educação superior; Ensino; Metodologia; Ciências da saúde.

\begin{abstract}
Objective: to report the experience of creating a conceptual map in face-to-face teaching during the coronavirus pandemic. Methodology: this is a descriptive-exploratory study of the type of experience report. Results: Both in the face-to-face and virtual class, the students showed total interest and willingness to participate in the proposed activities. In the first class held in person, students were able to expand their knowledge and integration using various tools encouraging the use of creativity through the use of pens, paints, colored pencils, promoting the interaction of students who at the end could share their work and perform a self assessment. In remote classes, virtual platforms were used to carry out the activity, also divided into groups, even though students could remotely exercise the integration dialogue. In this way, we can see that the use of concept maps together with the practice of active learning becomes positive both in the remote environment and in person, helping to understand concepts. Conclusion: We conclude that the use of the conceptual map in conjunction with active learning helps in the development of an educational environment both in person and in a pleasant virtual environment, capable of promoting integration, critical thinking and autonomy among students.
\end{abstract}

Keywords: Education higher; Teaching; Methodology; Health sciences.

\section{Resumen}

Objetivo: reportar la experiencia de crear un mapa conceptual en la docencia presencial durante la pande-mia de coronavirus. Metodología: se trata de un estudio descriptivo-exploratorio del tipo de relato de experiencia. Resultados: Tanto en el aula como en la clase virtual, los estudiantes mostraron total interés y disponibilidad para participar en las actividades propuestas. En la primera clase realizada presencialmente, los estudiantes pudieron ampliar sus 
conocimientos e integración utilizando diversas herramientas estimu-lando el uso de la creatividad mediante el uso de bolígrafos, pinturas, lápices de colores, promoviendo la interacción de los estudiantes que al final pudieron compartir su trabajo y realizar una autoevaluación. En las clases remotas se utilizaron plataformas virtuales para realizar la actividad, también divididas en gru-pos, aunque los estudiantes pudieron ejercitar de forma remota el diálogo de integración. De esta forma, podemos ver que el uso de mapas conceptuales junto con la práctica del aprendizaje activo se vuelve posi-tivo tanto en el entorno remoto como presencial, ayudando a comprender conceptos. Conclusión: Se con-cluye que el uso del mapa conceptual en conjunto con el aprendizaje activo ayuda en el desarrollo de un ambiente educativo tanto presencial como en un ambiente virtual agradable, capaz de promover la integra-ción, el pensamiento crítico y la autonomía entre los estudiantes.

Palabras clave: Educación superior; Enseñanza; Metodología; Ciencias de la salud.

\section{Introdução}

O uso do mapa conceitual como recurso tecnológico educacional é uma estratégia útil para consolidar a aprendizagem ativa que visa promover uma maior dinamicidade no ambiente de ensino (Dias et al., 2020). O recurso tecnológico emprega o uso de novas ferramentas didáticas no ambiente educacional visando o aperfeiçoamento da qualidade na transmissão do conhecimento. Com o aprimoramento da tecnologia surge também a necessidade da criação e melhorias de métodos que integrem o ambiente educacional e tecnológico, utilizando o mesmo como ferramenta positiva (Carneiro et al., 2020).

Para entendermos o ambiente educacional e suas dinâmicas precisamos focar na aprendizagem ativa que diferente da aprendizagem passiva onde o professor apenas deposita o conhecimento e o aluno o recebe de forma inativa, nele o aluno possui um papel ativo e participante se tornando assim protagonista na criação, desenvolvimento e resolução de atividades. Sendo assim o professor opera como facilitador criando uma ponte entre ensino e aprendizagem (Macedo et al., 2018).

A criação de mapas conceituais permite ao aluno a liberdade para distribuir, formar conceitos e integrá-los ao eixo principal criando uma estrutura para o conhecimento. Portanto os mapas conceituais são ferramentas gráficas que auxiliam na consolidação do tema estudado pelos estudantes, organizando de forma lógica e hierárquica os conteúdos aplicados, promovendo uma maior interação entre os alunos que podem vivenciar um trabalho em grupo. A criação de mapas conceituais é um recurso tecnológico que pode ser utilizado para avaliação da aprendizagem e como recurso didático-pedagógico na sala de aula presencial e também no ensino remoto, nas salas de aula virtuais. Para isso o aluno precisa estar atento aos elementos requisitados para a criação dos mesmos (Lima et al., 2017).

Com o avanço da pandemia provocada pelo Sars-Cov-2, agente etiológico da coronavirus disease 2019 (COVID-19) as instituições de ensino superior passaram por um período de mudanças que exigiram o cancelamento de suas atividades presenciais e requereram uma rápida adaptação à nova realidade (Lira et al., 2020; Costa et al., 2020).

Foram adotadas recursos e ferramentas tecnológicas educacionais que permitem a continuidade das atividades de ensino-aprendizagem. Estas mudanças e o uso de novas ferramentas trouxeram novos desafios aos gestores e professores na criação de estratégias de ensino que continuassem fornecendo uma formação de profissionais capazes, autônomos e responsáveis (Soares, Silva \& Moncaio, 2019).

O presente estudo objetivou relatar a experiência da criação de mapa conceitual no ensino presencial ao remoto durante a pandemia do coronavírus.

\section{Metodologia}

Trata-se de um estudo descritivo-explicativo do tipo relato de experiência que proporcionou a descrição das experiências da criação de mapa conceitual, realizadas no componente curricular de metodologia científica nos semestres letivos presenciais 2018.2 e 2019.2; e no remoto 2020.3, período letivo suplementar (PLS). O componente curricular é ofertado anualmente pelo colegiado do curso de graduação em enfermagem da Universidade de Pernambuco, campus Petrolina para alunos dos cursos de saúde: nutrição, fisioterapia e enfermagem. 
Enquanto componente obrigatório desses cursos, tem como competência compreender os fundamentos da metodologia científica integrando ensino, pesquisa e extensão. Este, promove a apresentação do mundo acadêmico para os calouros, tendo em vista que desponta nas grades curriculares dos primeiros períodos dos cursos, com carga horária de 45 horas, que contemplam aulas teóricas e mais uma hora semanal para o atendimento ao aluno.

O plano do componente curricular foi elaborado de modo a incentivar uma abordagem participativa, voltada a modelos educacionais mais inovadores que inspirassem os participantes. Adotou-se como estratégia didático-metodológica a aprendizagem ativa implementando-se ambientes educacionais que estimulassem a vontade de aprender junto com os colegas e professora em uma relação dialógica com o dia-a-dia, focado em competências transversais.

Neste sentido, optou-se por oferecer o componente organizado em quatro trilhas de aprendizagem com os seguintes conteúdos formativos: o problema do conhecimento, fundamentos da metodologia científica, ética na pesquisa, ensino e extensão, abordagens qualitativa e quantitativa, tipos de pesquisa, técnicas de estudo e planejamento do trabalho científico: resumos, desenvolvimento do tema, problema, problemática, objetivos, e normas da Associação Brasileira de Normas Técnicas (ABNT).

Destaca-se neste estudo, a terceira trilha, denominada "Abordagens de pesquisa e critérios de cientificidade na investigação", que tem proposto a cada semestre, tanto no ensino presencial quanto remoto, a criação de mapa conceitual com o objetivo de elaborar uma síntese do conhecimento sobre classificações, abordagens e recomendações para a elaboração de pesquisas científicas.

As estratégias, ferramentas e recursos para a criação participativa do mapa conceitual como tecnologia educacional difere de acordo com o contexto de ensino. Estas particularidades da implementação e as transformações que orientaram o componente curricular do ensino presencial ao remoto serão descritas nos resultados deste estudo.

\section{Resultados e Discussão}

No ensino presencial de metodologia científica, conteúdos formativos muito densos e repleto de conceitos, definições, regras e normas sobre a estrutura e elaboração de trabalhos acadêmicos e pesquisas científicas não costumam despertar o interesse e participação dos estudantes recém-chegados à Universidade. O professor enfrenta um desafio: tornar este aprendizado atrativo e prazeroso. Para tanto, optou-se por oferecer formas mais participativas de fazer o componente acontecer.

Neste contexto, na terceira trilha de aprendizagem, orientou-se a criação de um mapa conceitual. Ele funciona como uma representação gráfica do novo conhecimento aprendido e das transformações do conhecimento prévio sobre o tema, em uma colaboração constante entre alunos e professores (Machado \& Carvalho, 2019). Para fundamentar a construção do conhecimento disponibilizou-se, previamente, referencias teóricos e aportes didáticos como livros, artigos, link para sites e plataformas de pesquisa científica sobre o tema central: classificações, abordagens e recomendações para a elaboração de pesquisas científicas.

Os alunos deveriam reunir as informações e os conhecimentos prévios, de modo a exercitar o pensamento crítico e refletir sobre os diálogos nos encontros anteriores em sala de aula. Inclusive os conteúdos da primeira e segunda trilha de aprendizagem. Estimulou-se o uso da criatividade, propondo-se recursos como papeis, canetas e lápis coloridos, tintas, marcadores e fitas adesivas. No ensino presencial todos os recursos permitidos eram os físicos, o mapa deveria ser criado manualmente e na sala de aula.

A atividade de criação do mapa foi realizada em grupo. Como o objetivo era integrar os alunos e incentivar a colaboração, realizou-se sorteio entre os 40 alunos matriculados no componente curricular para a implementação de grupos com de quatro a seis participantes cada. 
Figura 1. Grupos criando o mapa conceitual (A) participantes sentados nas cadeiras e (B) no chão da sala de aula, PetrolinaPernambuco, Brasil, 2019,
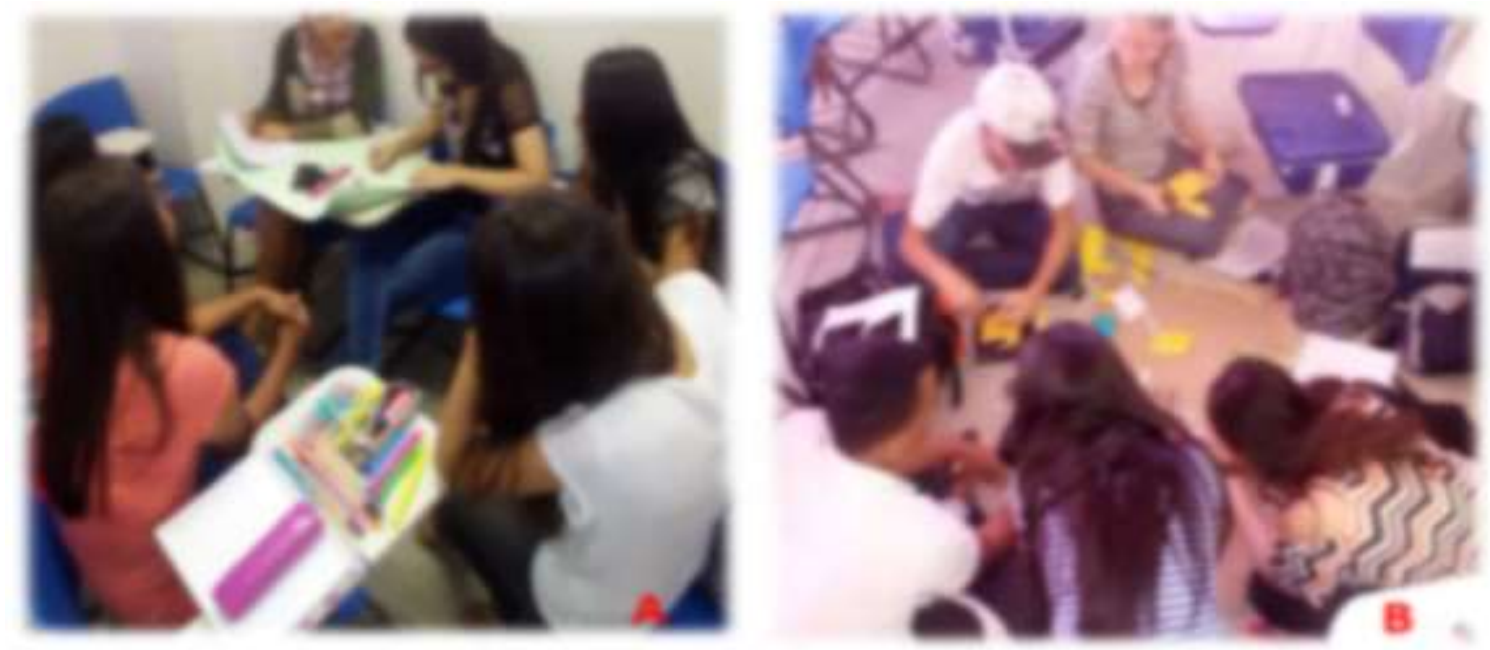

Fonte: Banco de imagens dos pesquisadores.

Como era esperado a interação entre os envolvidos foi positiva e mesmo não tendo sido solicitado todos optaram por se organizar em círculo, sentados nas cadeiras ou no chão, como mostra a imagem. Optamos por editar a figura inserindo o recurso artístico desfocar, para assegurar a preservação da imagem pessoal dos alunos (figura 1). A participação dos membros suscitou dúvidas sobre a posição e ordenamento das informações no mapa, bem como das relações entre os conceitos aprendidos até aquele momento nas trilhas que foram superadas com o diálogo entre estudantes e professora. Ao final da atividade os alunos autoavaliaram o desempenho do grupo e o desenvolvimento da atividade, revelando a consolidação da aprendizagem de conceitos importantes para a prática acadêmica.

Estudos sobre a utilização de mapa conceitual em sala de aula presencial revelam que o uso do mapa conceitual aliado a uma aprendizagem significativa auxilia os estudantes na organização de pensamentos transitando de um conhecimento mecânico para ativo. O uso do mapa conceitual também colabora para a formação de um profissional capaz de relacionar e interpretar dados favorecendo a organização do cuidado em saúde (Sousa et al., 2015; Salbego et al., 2018). Deste modo acredita-se que o uso de mapas conceituais pode auxiliar no desenvolvimento de uma consciência crítica, estimulando a criatividade e flexibilidade (Carvalho et al., 2016; Bittencourt et al., 2011).

Com a necessidade de distanciamento social, imposta pela COVID-19, em virtude das exigências sanitárias, iniciou-se a vivência do ensino remoto. Nele a terceira trilha de aprendizagem iniciou com uma webconferência de apresentação dos objetivos e conteúdos formativos e atividade avaliativa, que seria da criação do mapa conceitual com o tema central: classificação (tipos) de pesquisa.

Tratando-se de uma atividade avaliativa, a criação de cada um dos dois mapas conceituais teve peso total 5,0 pontos na nota da terceira trilha. Quando aos critérios para a distribuição da pontuação, criou-se as seguintes rubricas no google classroom $®:$ 1) conceitos claros; 2) relação justificada entre os conceitos; 3) riqueza de ideias; 4) criatividade na organização dos conceitos (utilização do dos recursos do Jamboard $($ ); e 5) representatividade do conteúdo no mapa, a cada um dos critérios seria atribuído até 1,0 ponto (Anastasiou, 2012).

Sobre o uso de mapas como ferramenta de avaliação, estudos têm apresentado perspectivas com critérios qualitativos, com ênfase no aumento da participação dos alunos e quantitativos, demonstrando aumento no aproveitamento com base no coeficiente global dos alunos (Dantas, Silva, \& Silva, 2018; Souza-Júnior et al., 2017).

Deste modo, para a criação do mapa conceitual na trilha, disponibilizou-se materiais de estudo no google Classroom® 
e em um momento da aula expositiva-dialogada síncrona, demonstrou-se a utilização do recurso de criação de notas adesiva (post-it digitais), o Jamboard ${ }^{\circledR}$. O acesso aos materiais de estudo e ao recurso interativo estava disponível com login pelo email institucional ao pacote $G$ Suíte®.

No encontro seguinte, durante a aula expositiva-dialogada realizada através do google meet ${ }^{\circledR}$, foi proposto aos alunos que se dividissem em grupos para construir o mapa conceitual, para tanto gerou-se link para duas novas salas de aula virtuais. Havia doze alunos matriculados, o que permitiu a criação de dois grupos, cada um com seis participantes. Os alunos produziram os mapas nas salas criadas e depois retornaram para a sala principal para a discussão entre ambos os grupos e professora.

O uso do recurso didático, mapa conceitual promoveu a participação ativa durante a aula expositiva-dialogada no ambiente virtual, rompendo a barreira da distância física. Esta possibilidade que o recurso didático propõe durante as aulas foi evidenciado também outros estudos (Queiroz et al., 2018; Tolfo, 2020).

Sobre os recursos do Jamboard®, destaca-se que é um ambiente para interagir com os colegas na criação de murais colaborativos (Virto \& Lopes, 2020), que nesta atividade avaliativa funcionou com um mapa conceitual virtual. Solicitou-se que utilizassem a criatividade com liberdade para inserir figuras, perguntas, post-it etc.

A utilização dessas tecnologias durante o ensino remoto possibilita a ampliação da aprendizagem. Pois, proporciona uma aprendizagem ativa, na qual o estudante é o protagonista na sala de aula e o criador do seu conhecimento, em contrapartida, ao modelo tradicional em que o estudante é o sujeito passivo que apenas recebe a informação (Jara \& Castro, 2017).

Apesar do avanço das metodologias de ensino-aprendizagem que visam suprir as necessidades dos estudantes, buscando estratégias que facilitem a construção do conhecimento, observa-se ainda uma dificuldade para os educadores utilizarem esses recursos. Isso em parte ocorre devido à ausência na formação e a limitação de conhecimento em relação às metodologias existentes (Santos et al., 2019).

Durante a trilha, produziu-se uma oficina sobre a confecção de mapa conceitual utilizando também o google docs ${ }^{\circledR}$, tendo em vista a proposta de atividade para os estudantes que foram convidados a elaborar um outro mapa conceitual, desta vez, sobre um dos tipos de estudo: coorte, ensaio clínico randomizado, caso controle, ecológico, revisão sistemática de literatura, revisão de escopo e estudo de validação. A Figura 2 a seguir demonstra mapas criados coletivamente no Jambord® durante encontro síncrono no google meet ${ }^{\circledR}$; e individualmente no google docs ${ }^{\circledR}$ e compartilhado no google drive ${ }^{\circledR}$. 
Figura 2. Mapa conceitual interativo criado no Jambord® (A) e Mapa conceitual individual criado no google docs®. (B), Petrolina-Pernambuco, Brasil, 2020.

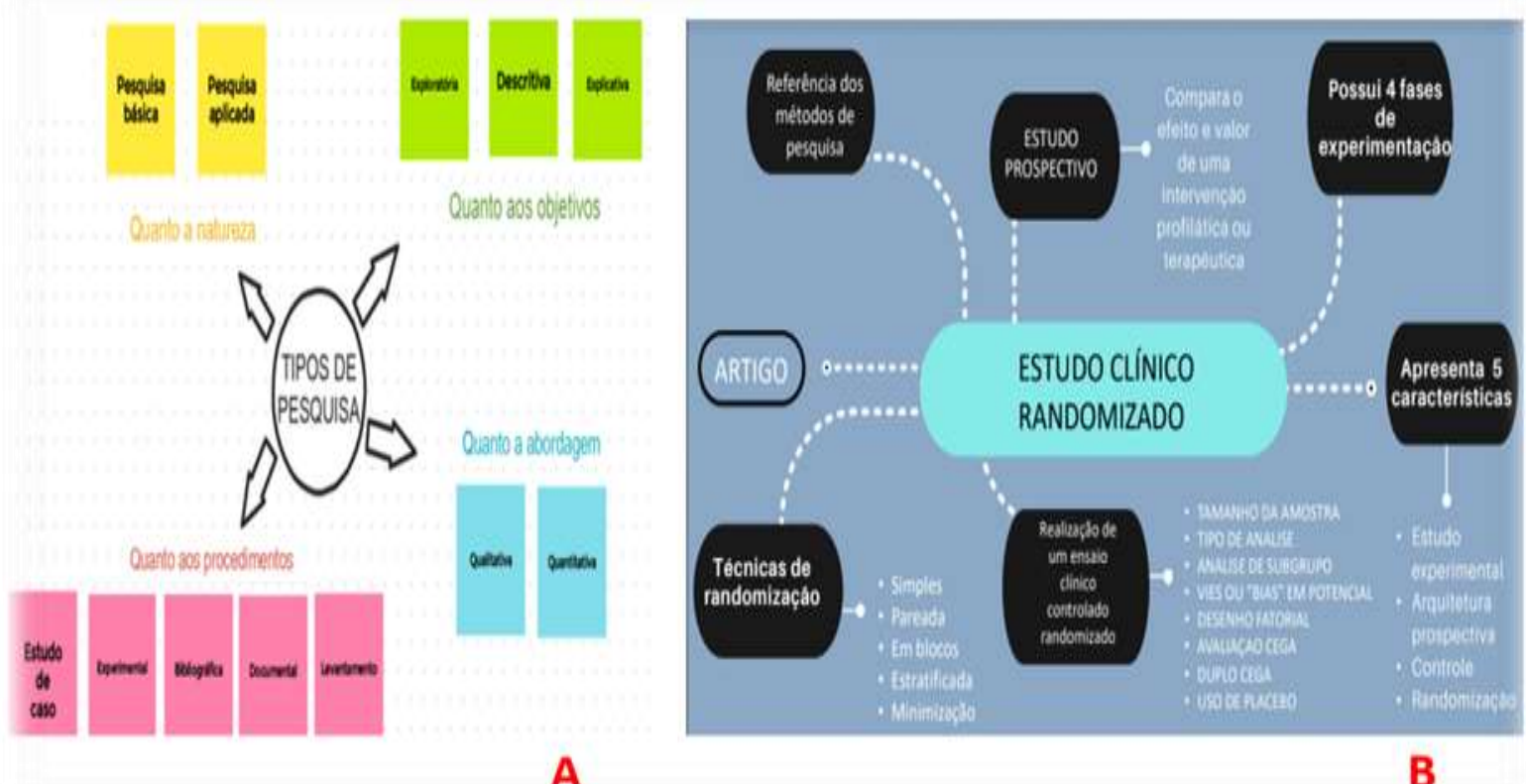

Fonte: Dados armazenados na nuvem (google drive $\left.{ }^{\circledR}\right)$ pesquisadores.

Cada aluno ficou responsável por postar, como atividade no google classroom ${ }^{\circledR}$, um artigo científico do tipo de estudo que ficou responsável e o mapa conceitual elaborado. No encontro síncrono seguinte, cada aluno apresentou os conceitos e características do tipo de estudo no mapa que elaborou. Os estudantes foram incentivados a interagir na apresentação dos colegas, fazendo perguntas e proposições.

Deste modo obteve-se a experiência da criação de mapa conceitual na interação com os colegas, fortalecendo o trabalho em equipe, bem como a elaboração de mapa como um exercício individual de pesquisa e do pensamento crítico. Exercitou-se também a apresentação das ideias em formato gráfico e oral e estimulou-se o debate e exemplificação dos conceitos estudados.

\section{Considerações Finais}

A experiência da criação de mapa conceitual no ensino presencial despertou vontade de aprender os conteúdos formativos entre os quatro grupos de alunos e tornou o momento do encontro em sala de aula, para discussão de temas tão densos e técnicos; prazeroso.

Sobre a criação no ensino remoto considera-se que ambos os grupos conseguiram usar o Jamboard®, demonstrando que a utilização desse recurso traz benefícios como aula mais didática, interação entre os alunos, e promove as habilidades de identificar informações de conteúdo aprendido previamente, avaliar e criar algo de maneira diferente. Enquanto, a utilização do google docs® permitiu a consolidação organizada e autônoma de novos conhecimentos de modo individual e facilitou o processo comunicativo entre os pares na apresentação oral do recurso.

\section{Referências}

Anastasiou, L. G. C. \& Alves L. P. (2012). Estratégias de ensinagem. In: Anastasiou, L. G. C. \& Alves L. P. Processos de ensinagem na universidade. Pressupostos para as estratégias de trabalho em aula. (10a ed.), Univille, 75-107. 
Bittencourt, G. K. G. D., Schaurich, D., Marini, M., \& Crossetti, M. G. O. (2011). Aplicação de mapa conceitual para identificação de diagnósticos de enfermagem. Rev bras enferm, 64(5), 963-967. https://doi.org/10.1590/S0034-71672011000500025

Carneiro, L. A., Rodrigues, W., França, G., \& Prata, D. N. (2020). Research, Society and Development, 9(8), e26798548. http://dx.doi.org/10.33448/rsdv9i8.5485

Carvalho, D. P. S. R. P., Vitor, A. F., Barichello, E., Villar, R. L. A., Pereira-Santos, V. E., \& Ferreira-Junior, M. A. (2016). Aplicação do mapa conceitual: resultados com diferentes métodos de ensino-aprendizagem. Aquichan, 16(3), 382-391. 10.5294/aqui.2016.16.3.92.

Conterno, S. F. R., \& Lopes, R. E. (2016). Pressupostos pedagógicos das atuais propostas de formação superior em saúde no Brasil: origens históric as e fundamentos teóricos. Avaliação (Campinas), http://www.scielo.br/scielo.php?script=sci_arttext\&pid=S1414-40772016000300993\&lng=en\&nrm=iso

Costa, R., Lino, M. M., Souza, A. I. J., Lorenzini, E, Fernandes, G. C. M., Brehmer, L. C. F., Vargas, M. A. O, Locks, M. O. H., \& Gonçalves, N. (2020). Ensino de enfermagem em tempos de covid-19: como se reinventar nesse contexto? Texto \& Contexto Enfermagem, 29(1), e20200202. https://doi.org/10.1590/1980-265x-tce-2020-0002-0002

Dantas, M. P., Silva, F. U., \& Silva-Borges, J. C. (2018). Uso dos mapas conceituais como ferramenta de avaliação qualitativa com ênfase no ensino de Física. HOLOS, 3(1),186-200. https://doi.org/10.15628/holos.2018.5932

Dias, I. C. B., Lima, E. F. A., Fioresi, M., Carrara, D., Oliveira, I. S., \& Primo, C. C. (2020). Construction of hypermedia for prevention of bloodstream infection. Rev Bras Enferm, 73(6), e20190593. https://doi.org/10.1590/0034-7167-2019-0593

Jara, V., \& Castro, J. (2017). Desarrollo de juicio clínico con mapas conceptuales de cuidado: experiencia de estudiantes de enfermería. Enfermería Univ, 14(4), 259-265. http://dx.doi.org/10.1016/j.reu.2017.09.003

Lima, J. A., Sampaio, C. de G., Barroso, M. C. S., Vasconcelos, A. K. P., \& Saraiva, F. A. (2017). Avaliação da aprendizagem em química com uso de mapas conceituais. Revista Thema, 14(2), 37-49. https://doi.org/10.15536/thema.14.2017.37-49.422.

Lira, A. L. B. C., Adamy-Edlamar, K., Teixeira, E., \& Silva, F. V. (2020) Educação em enfermagem: desafios e perspectivas em tempos da pandemia COVID19. Rev Bras Enferm, 73(Suppl2): e20200683. https://doi.org/10.1590/0034-7167-2020-0683

Macedo, K. D. S., Acosta, B. S., Silva, E. B., Souza, N. S., Beck, C. L., \& Silva, K. K. D. (2018). Metodologias ativas de aprendizagem: caminhos possíveis para inovação no ensino em saúde. Escola Anna Nery, 22(3), e20170435. https://dx.doi.org/10.1590/2177-9465-ean-2017-0435

Machado, C. T., \& Carvalho, A. A. (2019). Os efeitos dos mapas conceituais na aprendizagem dos estudantes universitários. Educação Temática Digital, 21(1), 259-277. https://doi.org/10.20396/etd.v21i1.8652010

Queiroz, I. R., Mendes, N. F., Almeida-Galdino, D. A., Silva-Amaral, J. H., \& Oliveira-Andrade, L. (2018). Metodologia Mapas Conceituais Aplicada ao Ensino Superior: uma Experiência com a Disciplina de Biologia Celular. Revista EducaOnline, 12(3), 69-87.

Salbego, C., Nietsche, E. A., Teixeira, E., Girardon-Perlini, N. M. O., Wild, C. F., \& Ilha, S. (2018). Care-educational technologies: an emerging concept of the praxis of nurses in a hospital context. Rev Bras Enferm, 71(Suppl 6): 2666-2674. https://doi.org/10.1590/0034-7167-2017-0753

Santos, L. S. F., Cama, A. C. L. F., Nogueira, G. A., Tavares, G. S., Rosa, A. F., \& Nunes, C. M. C. F (2017). Jogo da memória sobre feridas e curativos como estratégia de ensino aprendizagem. REAID, 83(21). https://doi.org/10.31011/reaid-2017-v.83-n.21-art.288

Soares, L. S., Silva, N. C., \& Moncaio, A. C. S. (2019). Metodologias Ativas no ensino superior: opiniões, conhecimentos e atitudes docentes. Rev enferm UFPE online,13(3),783-95. https://doi.org/10.5205/1981-8963-v13i03a236317p783-795-2019

Sousa, A. T. O., Formiga, N. S., Oliveira, S. H. S., Costa, M. M. L., \& Soares, M. J. G. O. (2015). Using the theory of meaningful learning in nursing education. Rev. Bras. Enferm. 68(4), 713-722. https://doi.org/10.1590/0034-7167.2015680420i

Souza Júnior, M. V., Célio, V. C. C., Nogueira, S. C. O., Martins, A. F., Freitas, K. H. G., \& Sousa, F. F. (2017). Mapas conceituais no ensino de física como estratégia de avaliação. Scientia Plena, 13(1),1-11. doi:http://dx.doi.org/10.14808/sci.plena.2017.012723

Tolfo, C. (2020). Concept Maps and promoting active classroom participation. Research, Society and Development, 9(1):e69911630. http://dx.doi.org/10.33448/rsd-v9i1.1630

Virto, N. R. \& López, M. F. B. (2020). Google Jam board Interactive Smart board: Are Innovative Approaches Useful in Personal Branding Assignments? $2^{o}$ Wold Conference on Future of Educacion. https://www.dpublication.com/wp-content/uploads/2019/09/4027-WCF.pdf 